\title{
Bond length order parameter of conjugated polymers
}

\author{
Chih-Ming Lai, Hsin-Fei Meng* \\ Institute of Physics, National Chiao Tung University, Hsinchu 300, Taiwan
}

Received 9 November 1998; accepted 4 February 1999

\begin{abstract}
It is usually assumed that the ground state lattice configuration of polyacetylene is a dimerized chain. We found that the energy can be further reduced by an overall chain length contraction, which leads to a new bond length order parameter, in addition to the well-known bond alternation order parameter. For quasi-particles like solitons and polarons, these two order parameters are coupled with the electron wave functions and vary in space. We generalize the Takayama-Liu-Maki equations in the continuum limit to incorporate this new order parameter. Significant modifications are found for the polaron solution. Polyacene, a ladder polymer, is also studied with the inclusion of the bond length order parameter. The ground state is predicted to be a non-alternating structure, with zero energy gap and unusually high electric conductivity. (C) 1999 Elsevier Science B.V. All rights reserved.
\end{abstract}

PACS: $71.20 . \mathrm{Hk}$

Keywords: Conjugated polymers; Solitons; Polarons

\section{Introduction}

Conjugated polymers are quasi-one-dimensional systems with electron and lattice degrees of freedom. In most theoretical studies [1,2], the lattice part is treated classically, i.e., within the adiabatic approximation. The lattice configurations of both ground state and the excited states are determined not only by the $\sigma$-bond elastic potential energy, but also by the coupling between the $\pi$-electrons and the lattice. Consider polyacetylene as an example. If there were no electron-lattice coupling, it would

\footnotetext{
*Corresponding author. Tel.: + 886-3-5731955; fax: + 8863-5720810.

E-mail address: meng@cc.nctu.edu.tw (H.-F. Meng)
}

have only one carbon atom per unit cell, and the total number of electrons would fill half of the single $\pi$-band, since there is one $\pi$-electron per carbon $p_{z}$ orbital. However, according to Peierls instability [3], a one-dimensional system with a half-filled band can lower its ground state energy by a dimerization with doubled unit cell. In other words, a uniform bond length becomes an alternation of short and long bonds, with their average (and therefore the total chain length) remaining the same. The question that whether the energy can be further lowered by a tripling or quadrupling of the unit cell still remains. This question is solved, more recently, by Kennedy and Lieb [4] with a rigorous proof that the dimerized state with doubled unit cell indeed has the lowest energy for a periodic lattice with the nearest-neighbor electron hopping integral varying linearly with the bond length. 
The purely dimerized structure is not, however, the most general form of lattice distortion caused by the coupling between the $\pi$-electrons and the lattice. An over all lattice contraction or stretch cannot be ruled out in the first place. In other words, it is possible that the total energy can be further lowered if, in addition to the alternation, the change of the average bond length is allowed. Simple calculation does show that the force exerted on each $\sigma$-bond by the $\pi$-electron coupling has a uniform part as well as an alternating part. Such force, therefore, cannot be completely counter balanced by a pure dimerization. In view of this, we introduced the second kind of order parameter: the bond length order parameter, in addition to the well-known bond alternation order parameter. These two bond order parameters (BOP) must be treated at equal footing in considering the lattice configurations and electronic structures for both the ground state and the quasi-particles. Through explicit calculation of the total ground state energy as a function of the BOPs, we found that there is indeed an overall bond length contraction due to the electron-lattice coupling. Therefore, for a given set of physical parameters, the electronic bandwidth and the band gap are different for the cases of including one and including two BOPs. The second BOP has a even more pronounced effect on the properties of the quasi-particles such as solitons, polarons and bipolarons. Both of the BOPs have non-uniform profiles around the quasi-particles. Because the BOPs are coupled to the electron wave functions through a set of self-consistent equations, the electron spectrum and wave functions are also changed significantly by the inclusion of the new bond length order parameter.

Another kind of conjugated polymer that illustrates the dramatic effect of the bond length order parameter is polyacene: the simplest ladder polymer. Because polyacene with a very large number of monomer units has not been synthesized, the magnitude of its band gap, if any, has been theoretically controversial. Most of the theoretical studies predict the existence of an energy gap in the range of $0.3-0.5 \mathrm{eV}$. We study the ground state of polyacene by including both of the two bond order parameters, and found that the dimerized configuration with a gap is unstable against the non- alternating configuration. The consequence is that the finite band gap disappears in the true ground state configuration. We therefore predict that polyacene is a gapless semiconductor, for which the thermally excited carriers dominate doping at most temperatures. DC conductivity is expected to be much higher than other conjugated conducting polymers. We made explicit calculations and found that, in addition to being large, the conductivity has an unusual temperature and doping dependency as compared with other more typical conjugated polymers like polyacetylene.

This paper is organized as follows. In Section 2, we obtain the true ground state of trans-polyacetylene by minimizing the total energy with respect to the two uniform order parameters. In Section 3, the self-consistency equations in the continuum limit for the quasi-particles are derived. The intra-gap energy levels and the BOP profiles of solitons and polarons are obtained by solving the continuum equations numerically. The case of non-degenerate ground state polymer is also discussed. In Section 4 , the second BOP is applied to polyacene. We make a summary and conclude in Section 5.

\section{New bond order parameter and true ground state}

We start with the Su-Schrieffer-Heeger (SSH) model [5] with the Hamiltonian

$$
\begin{aligned}
H_{\mathrm{SSH}}= & -\sum_{l, s}\left[t_{0}-\alpha\left(u_{l+1}-u_{l}\right)\right] \\
& \times\left(c_{l+1, s}^{\dagger} c_{l, s}+c_{l, s}^{\dagger} c_{l+1, s}\right) \\
& +\sum_{l} \frac{K}{2}\left(u_{l+1}-u_{l}\right)^{2} .
\end{aligned}
$$

It is the simplest Hamiltonian that satisfies the Kennedy-Lieb criterion [4]. Here $c_{l, s}^{\dagger}$ and $c_{l, s}$ are the creation and annihilation operators of electrons at lattice site $l$ with spin $s . u_{l}$ is the displacement of the carbon atom at site $l$. The constants $t_{0}, \alpha, K$, are the electron hopping integral, electron-lattice coupling, and lattice elasticity, respectively. As discussed in Section 1, this model is usually solved with the dimerization ansatz. $u_{l+1}-u_{l}=(-1)^{l} 2 u$. the bond alternation order parameter $u$ is a 
constant for the ground state. Such a lattice distortion keeps the total chain length unchanged. Now we go beyond the ansatz and allow the lattice constant of the new unit cell, with one short bond and one long bond, to be different from twice of the original unit cell. The difference is denoted as $2 w$. In other words, the lattice can be stretched $(w>0)$ or contracted $(w<0)$. The dimerization ansatz is replaced by

$u_{l+1}-u_{l}=2 w+(-1)^{l} 2 u$.

The Hamiltonian in Eq. (1) becomes

$$
\begin{aligned}
H= & -\sum_{l, s}\left[t_{0}-2 \alpha w-(-1)^{l} 2 \alpha u\right] \\
& \times\left(c_{l+1, s}^{\dagger} c_{l, s}+c_{l, s}^{\dagger} c_{l+1, s}\right) \\
& +\frac{K}{2} \sum_{l}\left(4 w^{2}+4 u^{2}\right) .
\end{aligned}
$$

One can easily get the ground state energy $E_{0}[5]$

$E_{0}=-\frac{4 N}{\pi} t^{\prime} E\left(1-z^{\prime 2}\right)+\frac{K N}{2}\left(4 u^{2}+4 w^{2}\right)$,

with $t^{\prime} \equiv t_{0}-2 \alpha w_{0}$ and $z^{\prime} \equiv 2 \alpha u_{0} / t^{\prime} . N(\gg 1)$ is the total number of carbon atoms in the chain. For convenience, we rewrite $E_{0}$ in terms of the dimensionless variables.

$$
\begin{aligned}
\bar{\varepsilon}_{0}(x, y) & \equiv \frac{E_{0}(x, y)}{N} \\
& =-\frac{4 t_{0}}{\pi}\left[(1-y) E\left(1-z^{2}\right)-\frac{1}{4 \lambda}\left(x^{2}+y^{2}\right)\right] .
\end{aligned}
$$

Here $E\left(1-z^{2}\right)$ is the elliptic integral. $\lambda, x, y$, and $z$ are dimensionless parameters defined as $\lambda \equiv$ $2 \alpha^{2} /\left(\pi t_{0} K\right), x \equiv 2 \alpha u / t_{0}, \quad y \equiv 2 \alpha w / t_{0}, \quad$ and $\quad z \equiv$ $x /(1-y)$. The ground state values of $u$ and $w$ can be obtained by minimizing $E_{0}(x, y)$ with respect to $x$ and $y$. If $x, y \ll 1$ (i.e. $z \ll 1$ ), the elliptic integral can be expanded as

$$
E\left(1-z^{2}\right) \simeq 1+\frac{z^{2}}{2} \ln \frac{4}{|z|}-\frac{z^{2}}{4},
$$

and the total energy per electron $\bar{\varepsilon}_{0}$ becomes

$$
\begin{aligned}
& \bar{\varepsilon}_{0}(x, y) \\
& =\frac{4 t_{0}}{\pi}\left(C_{1}(y) x^{2} \ln \frac{4}{|x|}+C_{2}(y) x^{2}+C_{3}(y)\right),
\end{aligned}
$$

with

$$
\begin{aligned}
& C_{1}(y)=-\frac{1}{2(1-y)}, \\
& C_{2}(y)=-\frac{1}{2(1-y)} \ln |1-y|+\frac{1}{4 \lambda}+\frac{1}{4(1-y)}, \\
& C_{3}(y)=-(1-y)+\frac{y^{2}}{4 \lambda} .
\end{aligned}
$$

Through the minimal energy condition

$\frac{\partial \bar{\varepsilon}_{0}}{\partial x}=0, \quad \frac{\partial \bar{\varepsilon}_{0}}{\partial y}=0$,

we find the solutions $x_{0}$ and $y_{0}$

$y_{0}=-2 \lambda$,

$x_{0}=4 \exp \left[-\left(\frac{1}{2 \lambda}+1\right)+\left(\frac{1}{2 \lambda}-\frac{1}{2}\right) y_{0}\right]$.

The corresponding ground state BOPs are

$w_{0}=-\frac{t_{0}}{\alpha} \lambda$,

$u_{0}=\frac{2 t_{0}}{\alpha} \exp \left[-\left(\frac{1}{2 \lambda}+2-\lambda\right)\right]$.

The conventional result of the SSH model without $w$ can be reproduced if we set $w=0(y=0)$ in Eq. (7). The second kind BOP $w_{0}$ is in general not zero as long as there is an electron-phonon coupling. The negative $w_{0}$ implies the whole chain is contracted. The band gap $E_{\mathrm{g}}$ also depends on $w_{0}$. The mathematical relations between observable quantities (band gap and bandwidth) and the model parameters $\left(t_{0}, \alpha, K\right)$ are thus corrected by the second BOP. So far there would be no difference in experimental predictions with or without $w_{0}$ if we consider only the ground state, because the effect of the constant $w_{0}$ is equivalent to redefining a new electron hopping integral $t^{\prime} \equiv t_{0}-2 \alpha w_{0}$. The value of $t^{\prime}$ is obtained, after all, by fitting the 
experimental spectrum. However, many new interesting features arise if we consider the quasi-particles, in which $w$ and $u$ in Eq. (2) do vary in space. So we need to make the generalizations $u \rightarrow u(l)$ and $w \rightarrow w(l)$. They cause "bag-like" or "kink-like" variations not only in $u(l)$ but also in $w(l)$. This features are the subject of the next section.

\section{Continuum equations for quasi-particles}

In order to obtain the lattice configurations and the electron wave functions associated with the quasi-particles, we take the continuum limit for convenience. Takayama et al. [6] derived a set of continuum equations which couple the electron wave functions and the first BOP $u(l)$ self-consistently. We generalize the TLM equations in order to incorporate the second BOP $w(l)$. The single electron wave function $\left|\Psi_{k}\right\rangle$ is expanded as

$\left|\Psi_{k}\right\rangle=\sum_{l} \psi_{l, k}|l\rangle$

$|l\rangle$ is the carbon $p_{z}$ orbital at site $l$. We start with the eigenvalue equation for $\psi_{l, k}$

$\varepsilon_{k} \psi_{l, k}+t\left(r_{l, l+1}\right) \psi_{l+1, k}+t\left(r_{l, l-1}\right) \psi_{l-1, k}=0$.

$r_{l, l+1}$ is the bond length between site $l$ and $l+1$, and $t\left(r_{l, l+1}\right)$ is the hoping integral. In terms of the BOPs, $t\left(r_{l, l+1}\right)$ is expressed as $t\left(r_{l, l+1}\right) \equiv$ $t_{0}-\Omega_{l}-\frac{1}{2}(-1)^{l} \Delta_{l}, \quad$ with $\quad \Omega_{l} \equiv 2 \alpha w(l) \quad$ and $\Delta_{l} \equiv 4 \alpha u(l)$. We seek solution of the form $\psi_{l, k}=\mathrm{e}^{\mathrm{i} k l}\left[u_{k}(l)+(-1)^{l} i v_{k}(l)\right], u_{k}(l)$ and $v_{k}(l)$ are slowly varying functions of $l$. Given the occupation numbers $n_{k}$, the total energy $E$ is given by

$E=\sum_{l} V\left(r_{l, l+1}\right)+\sum_{k} \varepsilon_{k} n_{k}$

The self-consistent equation for the BOPs are determined by the minimal condition $\partial E / \partial r_{l, l+1}=0$. It can be easily shown that

$\frac{\partial \varepsilon_{k}}{\partial r_{l, l+1}}=-\frac{\mathrm{d} t\left(r_{l, l+1}\right)}{\mathrm{d} r_{l, l+1}}\left(\psi_{l, k}^{*} \psi_{l+1, k}+\psi_{l+1, k}^{*} \psi_{l, k}\right)$
From Eqs. (12) and (13), the minimum condition becomes

$\frac{\mathrm{d} V}{\mathrm{~d} r_{l, l+1}}=\frac{\mathrm{d} t}{\mathrm{~d} r_{l, l+1}} \sum_{k}\left(\psi_{l, k}^{*} \psi_{l+1, k}+\psi_{l+1, k}^{*} \psi_{l, k}\right) n_{k}$.

The is actually the balanced force condition. The RHS is the force exerted on the $\sigma=$ bond between site $l$ and $l+1$ by the $\pi$-electron coupling. It has in general a uniform part in addition to an alternating part. More specifically, it is of the form $a+(-1)^{l} b$. A purely dimerized structure, with the LHS proportional to $(-1)^{l}$, can never satisfy the balanced force condition. Substituting

$\frac{\mathrm{d} V}{\mathrm{~d} r_{l, l+1}}=\frac{K}{\alpha}\left(\Omega_{l}+\frac{1}{2}(-1)^{l} \Delta_{l}\right), \quad \frac{\mathrm{d} t}{\mathrm{~d} r_{l, l+1}}=-\alpha$

and

$\psi_{l, k}=\mathrm{e}^{\mathrm{i} k l}\left[u_{k}(l)+(-1)^{l} i v_{k}(l)\right]$

into Eqs. (11), (14) and taking the continuum limit $l a \rightarrow x, u_{k}(l) \rightarrow \sqrt{a} u_{k}(x), \Delta_{l} \rightarrow \Delta(x)$, and

$$
\begin{aligned}
u_{k}(l+1) & \rightarrow \sqrt{a} u_{k}(x+a) \\
& \simeq \sqrt{a}\left(u_{k}(x)+a \frac{\partial u_{k}(x)}{\partial x}\right), \\
v_{k}(l+1) & \rightarrow \sqrt{a} v_{k}(x+a) \\
& \simeq \sqrt{a}\left(v_{k}(x)+a \frac{\partial v_{k}(x)}{\partial x}\right),
\end{aligned}
$$

we obtain the following continuum equations

$$
\begin{aligned}
\varepsilon_{k} u_{k}(x)= & -\mathrm{i} 2 a t_{0} \frac{\partial u_{k}(x)}{\partial x} \\
& +\Delta(x) v_{k}(x)-\frac{a \partial \Delta(x)}{2} v_{k}(x) \\
& +\mathrm{i}\left[2 a \Omega(x) \frac{\partial u_{k}(x)}{\partial x}+a \frac{\partial \Omega(x)}{\partial x} u_{k}(x)\right], \\
\varepsilon_{k} v_{k}(x)= & \mathrm{i} 2 a t_{0} \frac{\partial v_{k}(x)}{\partial x}+\Delta(x) u_{k}(x)-\frac{a \partial \Delta(x)}{2} \frac{\partial x}{\partial x} u_{k}(x) \\
& -\mathrm{i}\left[2 a \Omega(x) \frac{\partial v_{k}(x)}{\partial x}+a \frac{\partial \Omega(x)}{\partial x} v_{k}(x)\right],
\end{aligned}
$$




$$
\begin{aligned}
\Omega(x)= & \frac{a \alpha^{2}}{K} \sum_{k}\left[\mathrm { i } \left(a \frac{\partial u_{k}^{*}(x)}{\partial x} u_{k}(x)\right.\right. \\
& \left.\left.-a \frac{\partial v_{k}^{*}(x)}{\partial x} v_{k}(x)\right)+c . c .\right] n_{k}, \\
\Delta(x)= & -\frac{4 a \alpha^{2}}{K} \sum_{k}\left[\left(u_{k}^{*}(x) v_{k}(x)+v_{k}^{*}(x) u_{k}(x)\right)\right. \\
& \left.+\frac{a}{2} \frac{\partial}{\partial x}\left(u_{k}^{*}(x) v_{k}(x)+v_{k}^{*}(x) u_{k}(x)\right)\right] n_{k} .
\end{aligned}
$$

In order to arrive at those equations, we have to identify both the average parts (e.g. $\Omega_{l}$ ) and the alternation parts (e.g. $\left.(-1)^{l} \Delta_{l}\right)$ of both sides of Eqs. (11) and (14). Since all the functions $u_{k}(x), v_{k}(x)$, $\Delta(x)$ and $\Omega(x)$ are assumed to be slowly varying, higher order terms like $\left(\partial^{2} / \partial x^{2}\right) u_{k}(x)$, $\left(\partial^{2} / \partial x^{2}\right) v_{k}(x)\left(\partial^{2} / \partial x^{2}\right) \Delta(x)$, and $\left(\partial^{2} / \partial x^{2}\right) \Omega(x)$ are neglected. When $\Omega(x)$ is set to zero, Eqs. (18), (19), and (21) reproduce the TLM continuum equations. Since Eqs. (18)-(21) cannot be solved exactly, we make iterations and choose TLM's exact solutions $\left\{\tilde{u}_{k}, \tilde{v}_{k}, \tilde{\Delta}(x)\right\}[6,7]$ as the starting point. First, we substitute $\tilde{u}_{k}$ and $\tilde{v}_{k}$ into Eqs. (20) and (21) to find a new $\tilde{\Omega}_{1}(x)$ and $\tilde{\Delta}_{1}(x)$. Then we substitute $\tilde{\Omega}_{1}(x), \tilde{\Delta}_{1}(x)$ back into the electron wave function Eqs. (18) and (19) to find new $\tilde{u}_{1 k}, \tilde{v}_{1 k}$ and then bring them back into Eqs. (20) and (21) to find $\tilde{\Omega}_{2}(x)$ and $\tilde{\Delta}_{2}(x) \ldots$, and so on. Of course, the normalization condition for the wave functions $u_{k}$ and $v_{k}$ are kept through out the iteration.

Before presenting the quasi-particle solutions, we first consider the ground state, for which both of the BOPs are uniform and the solution is trivial. For the discrete Hamiltonian in Section 2, we have $\Omega_{0} \equiv 2 \alpha w_{0}=-2 t_{0} \lambda$. Using the parameters $t_{0}=2.3 \mathrm{eV}, K=20 \mathrm{eV} / \AA^{2}$, and $\alpha=4.55 \mathrm{eV} / \AA$, we have $\Omega_{0}=-1.3 \mathrm{eV}$. In the continuum approximation, $\Omega_{0}=-1.03 \mathrm{eV}$ is obtained for the ground state using the same set of parameters. It is close to the discrete model, which indicates that the continuum limit is a reasonable approximation. Because it is the band gap that is actually observable, we use a slightly different set of parameters below for the continuum limit in order to fit the half band gap $\Delta_{0}$ of $0.7 \mathrm{eV}$. The values we use are $t_{0}=3 \mathrm{eV}$, $K=20 \mathrm{eV} / \AA^{2}$, and $\alpha=4.45 \mathrm{eV} / \AA$.
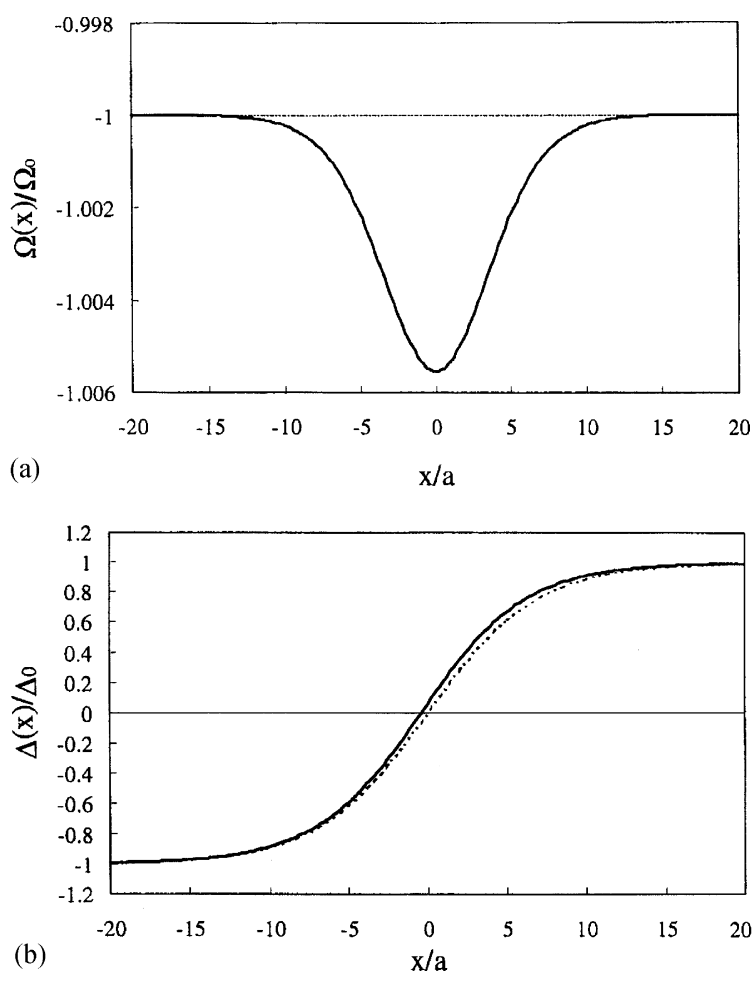

Fig. 1. (a) The second kind of BOP (bond length contraction) is shown for a soliton located at $x=0$. The dotted thin line is the constant value $\Omega_{0}$ for the ground state. The physical parameters are chosen as $t_{0}=3, \alpha=4.49 \mathrm{eV} / \AA$, and $K=20 \mathrm{eV} / \AA^{2}$. (b) The bond alternation order parameter $\Delta(x)$ (solid line) for a soliton is shown. The dotted line denotes $\Delta(x)$ without the inclusion of $\Omega(x)$ (TLM model). This figure shows a slight central-symmetry breaking of $\Delta(x)$.

In Figs. 1(a) and (b), $\Delta(x)$ and $\Omega(x)$ for a soliton are shown. $\Delta_{0}$ and $\Omega_{0}$ are their ground state values. The fact that the variation is small can be understood as follows. The charge-conjugate symmetry [7] is an important feature of the TLM model. It means that for every positive energy level $\varepsilon_{+}$with solution $\{u, v\}$, there is a negative energy level $\varepsilon_{-}=-\varepsilon_{+}$with solution $\{\mathrm{i} v,-\mathrm{i} u\}$. Since the existence of $\Omega(x)$ does not change the charge-conjugate symmetry of the original equations in the TLM model, both the continuous band and discrete intra-gap levels still keep the mirror symmetry with respective to the Fermi level at zero energy. In solitons, there is only one intra-gap level located at the Fermi level and with the second BOP it is 
impossible to cause any energy shift to this intragap level. One can then imagine that the local lattice structure around the center of the soliton will not have a significant change. Clearly such constraint does not apply to polarons which have two intra-gap levels symmetrically located at the opposite sides of the Fermi level. Figs. 2(a) and (b) show the BOPs for a polaron. As expected, the changes of BOPs in the polarons are much larger than that in the solitons. The inclusion of $\Omega(x)$ can cause energy shifts to these two levels without breaking their mirror-symmetry. We find that the intra-gap level above the Fermi level moves upward to the conduction band, while the other level below the Fermi level moves downward to the valence band. The magnitude of the energy shift of $\varepsilon_{+}\left(\varepsilon_{-}\right)$is $0.034 \mathrm{eV}(-0.034 \mathrm{eV})$, i.e., about $7 \%$ change compared with TLM model without $\Omega(x)$. It is also seen in Fig. 2(b) that $\Delta(x)$ of a polaron loses the central inverse symmetry. This is no surprise because it can be seen in Eq. (21), where the spatial symmetry of the derivative part on the right-hand side is opposite to the first part.

For the non-degenerate ground state conjugated polymers with an extrinsic hopping alternation such as cis-polyacetylene, the Hamiltonian is

$$
\begin{aligned}
H= & -\sum_{n, s}\left[t_{0}-2 \alpha w-(-1)^{n}\left(\frac{t_{\mathrm{e}}}{2}+2 \alpha u\right)\right] \\
& \times\left(c_{n+1, s}^{\dagger} c_{n, s}+c_{n, s}^{\dagger} c_{n+1, s}\right)+\frac{K}{2} \sum_{n}\left(4 w^{2}+4 u^{2}\right),
\end{aligned}
$$

with $t_{0} \equiv\left(t_{1}+t_{2}\right) / 2 . t_{\mathrm{e}} \equiv t_{1}-t_{2}\left(\right.$ say, $\left.t_{1}>t_{2}\right)$ is the extrinsic hoping alternation parameter. The total energy per electron $\bar{\varepsilon}_{0}(u, w)$ of the ground state can be readily obtained from Eq. (4) by replacing $z \rightarrow z^{\prime}=\left(x+t_{\mathrm{e}} / 2 t_{0}\right) /(1+y)$ :

$$
\begin{aligned}
\bar{\varepsilon}_{0}(x, y)= & \frac{4 t_{0}}{\pi}\left[-(1-y) E\left(1-z^{\prime 2}\right)+\frac{1}{4 \lambda}\left(x^{2}+y^{2}\right)\right] \\
\simeq & \frac{4 t_{0}}{\pi}\left[D_{1}(y)\left(x+\frac{t_{\mathrm{e}}}{2 t_{0}}\right)^{2} \ln \left|x+\frac{t_{\mathrm{e}}}{2 t_{0}}\right|\right. \\
& +D_{2}(y)\left(x+\frac{t_{\mathrm{e}}}{2 t_{0}}\right)^{2}+D_{3}(y)\left(x+\frac{t_{\mathrm{e}}}{2 t_{0}}\right) \\
& \left.+D_{4}(y)\right],
\end{aligned}
$$
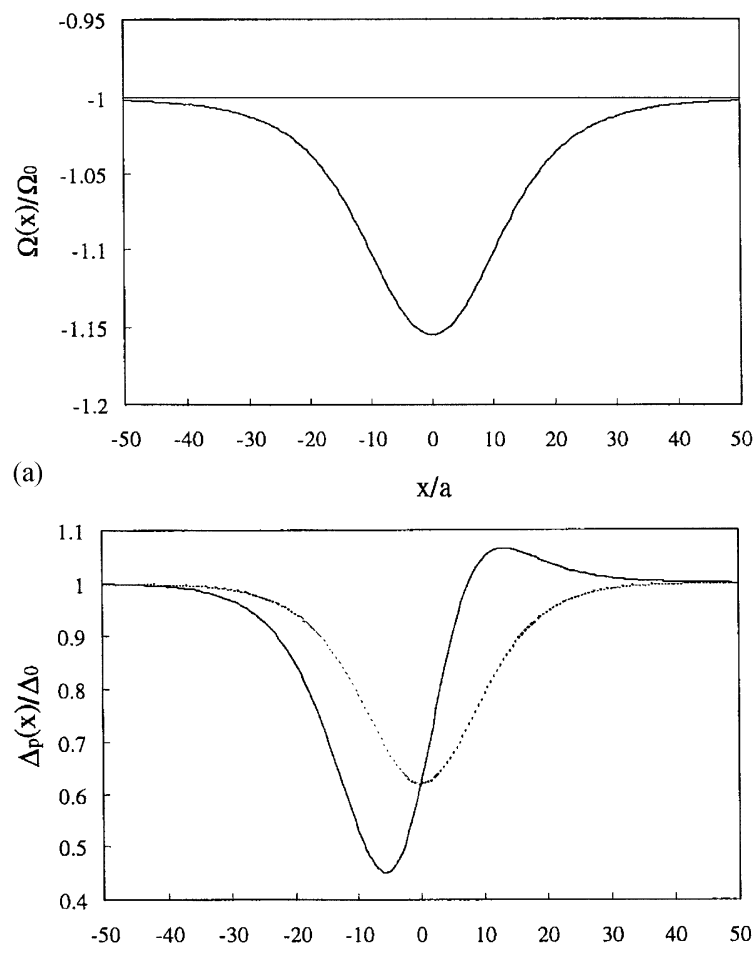

(b) $\mathrm{x} / \mathrm{a}$

Fig. 2. Similar to Fig. 1, we show the bond length order parameter (a) and the alternation order parameter (b) for a polaron. The dotted line in (b) is the usual polaron profile in the TLM model.

with

$$
\begin{aligned}
& D_{1}(y)=-\frac{1}{2(1-y)}, \\
& D_{2}(y)=-\frac{1}{2(1-y)} \ln 4|1-y|+\frac{1}{4 \lambda}+\frac{1}{4(1-y)}, \\
& D_{3}(y)=-\frac{t_{\mathrm{e}} / 2 t_{0}}{2 \lambda}, \\
& D_{4}(y)=-(1-y)+\frac{y^{2}}{4 \lambda}+\frac{\left(t_{\mathrm{e}} / 2 t_{0}\right)^{2}}{4 \lambda} .
\end{aligned}
$$

Minimizing the ground state energy as in Section 2, we get similar results that the chain is contracted as well as dimerized. The parameters $t_{0}=2.5 \mathrm{eV}$, $\alpha=4.63 \mathrm{eV} / \AA$ A $K=21 \mathrm{eV} / \AA^{2}$, and $t_{\mathrm{e}}=0.17 \mathrm{eV}$ are adopted to fit the band gap of $2.05 \mathrm{eV}^{2}$ for cispolyacetylene. The energy minimum is found to be at $u_{0}=0.046 \AA$, and $w_{0}=-0.138 \AA$. In the continuum limit, we can follow the same steps in 
Section 2 to get the self-consistent equations similar to Eqs. (18) and (19), except the replacement of $\Delta(x)$ by $\Delta_{\mathrm{p}}(x)=\Delta_{\mathrm{i}}(x)+\Delta_{\mathrm{e}}$. The intrinsic part $\Delta_{\mathrm{i}}(x)$ satisfies precisely the same relation as Eq. (21), and the extrinsic part $\Delta_{\mathrm{e}}$ is equal to $t_{\mathrm{e}}$. The self-consistent equation of $\Omega(x)$ is the same as Eq. (20).

\section{Conjugated ladder polymer}

Ladder polymers are conjugated polymers with at least two conduction paths in parallel. They share many of the properties with the polymers with a single conjugation path. In particular, many of the ladder polymers support polarons and bipolarons [8]. Polyacene is the simplest ladder polymer. The polyacene chain, with two identical backbone in parallel, can be viewed as two strongly interacting chains of trans-polyacetylene (see Fig. 3(a)). Polyacene is yet to be synthesized as a polymer with a large number monomer unit [9]. The electronic structure of polyacene has been the ob-

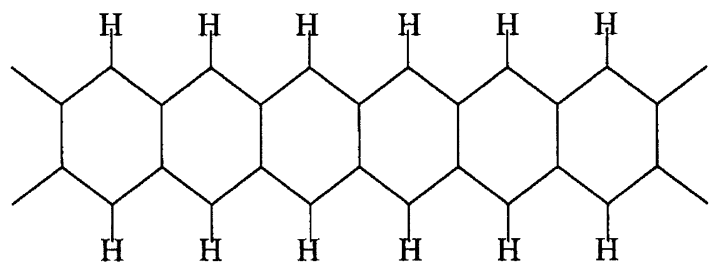

(a)

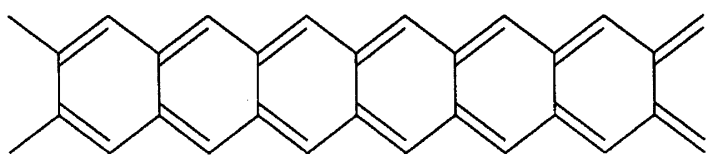

(b)

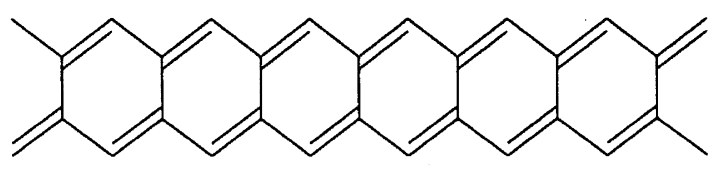

(c)

Fig. 3. (a) The chemical structure of polyacene. There is one carbon atom at each vertex. (b) Parallel ordered dimerization configurations. (c) Anti-parallel ordered dimerization configuration. ject of many theoretical studies [10-13]. Due to the lack of direct experimental evidences, there is some controversy about the existence and the size of the energy gap due to bond alternation. Among the works that support its existence, the predicted values of the gap have been in the range of $0.3-$ $0.5 \mathrm{eV}$.

In this section, we study the electronic structure of polyacene, with both of the two bond order parameters (alternation and contraction) taken into account in the search for the true ground state configuration. We predict that the dimerized configuration is unstable against a non-alternating configuration. The result is a gapless band structure, contrary to most of the previous theoretical works.

\subsection{Gapless band structure}

Let us consider a single chain of polyacene consisting of $2 \mathrm{~N}$ sites. It can be treated as two interacting chains of polyacetylene. Each chain of polyacetylene is described by the SSH Hamiltonian:

$$
\begin{aligned}
H_{j}= & -\sum_{n}\left[t_{\|}+(-1)^{n} \alpha\left(u_{j, n+1}-u_{j, n}\right)\right] \\
& \times\left(c_{j, n+1}^{\dagger} c_{j, n}+\text { h.c. }\right) \\
& +\frac{K}{2} \sum_{n}\left(u_{j, n+1}-u_{j, n}\right)^{2}
\end{aligned}
$$

where $j=1,2$ denotes the chain index. The interchain hopping is described by

$H_{\perp}=-\sum_{n} t_{\perp}\left[c_{1, n}^{\dagger} c_{2, n}+\right.$ h.c. $]$.

Here $u_{j, n}$ are the displacement coordinates and $c_{j, n}$ the annihilation operator at the $n$th lattice site on the $j$ th chain. For simplicity, spin indices are omitted. The interchain hopping integral $t_{\perp}$ is of the form

$t_{\perp}=\frac{1}{2}\left[t_{1}+(-1)^{n} t_{2}\right]$,

with $t_{1}=t_{2}$ for the case of polyacene. Now we look for the ground state configuration of polyacene by minimizing the total energy, with the ansatz that $u_{j, n+1}-u_{j, n}=w+(-1)^{n} u_{j}$. The reason that we allow $u$ to be $j$-dependent will be clear later. The 
Hamiltonian becomes

$$
\begin{aligned}
H= & \sum_{j, n}\left[-\left(t_{0}-2 \alpha w\right)+(-1)^{n} 2 \alpha u_{j}\right] \\
& \times\left(c_{j, n}^{\dagger} c_{j, n+1}+\text { h.c. }\right) \\
& +\sum_{n}-\frac{1}{2} t_{0}\left[1+(-1)^{n}\right]\left(c_{1 n}^{\dagger} c_{2 n}+\text { h.c. }\right) \\
& +\sum_{j, n} \frac{K}{2}\left[\left(2 u_{j}\right)^{2}+(2 w)^{2}\right] .
\end{aligned}
$$

One can introduce the annihilation operators $a_{j k}$ and $b_{j k}$ for electrons belonging to the conduction and valence bands, respectively, through the relation

$$
\begin{aligned}
c_{j, n} & =\frac{1}{\sqrt{N}} \sum_{k} \mathrm{e}^{\mathrm{i} k n a}\left[(-1)^{n} a_{j k}+\mathrm{i} b_{j k}\right], \\
& -\pi / 2 a<k<\pi / 2 a .
\end{aligned}
$$

Then $H_{j}$ can be diagonized by the Bogoliubov transformation

$a_{j k}=\cos \theta_{j k} \alpha_{j k}+\sin \theta_{j k} \beta_{j k}$,

$b_{j k}=\cos \theta_{j k} \beta_{j k}-\sin \theta_{j k} \alpha_{j k}$,

provided that $\tan \left(2 \theta_{j k}\right)=-\Delta_{j}\left(2 t_{0}\right)^{-1} \tan (k a)$, with $\Delta_{j}=4 \alpha u_{j}$. We then have

$$
H_{j}=\sum_{k} E_{k}\left(\alpha_{j k}^{\dagger} \alpha_{j k}-\beta_{j k}^{\dagger} \beta_{j k}\right),
$$

and $H_{\perp}$ becomes

$$
\begin{aligned}
H_{\perp}= & -\frac{t_{1}}{2} \sum_{k}\left\{\left[\left(\cos \left(\theta_{1 k}-\theta_{2 k}\right)+\mathrm{i} \sin \left(\theta_{1 k}-\theta_{2 k}\right)\right)\right.\right. \\
& \left.\times\left(\alpha_{1 k}^{\dagger} \alpha_{2 k}+\beta_{1 k}^{\dagger} \beta_{2 k}\right)+\text { h.c. }\right] \\
& +\left[\left(-\sin \left(\theta_{1 k}-\theta_{2 k}\right)+\mathrm{i} \cos \left(\theta_{1 k}-\theta_{2 k}\right)\right)\right. \\
& \left.\left.\left.\times\left(\alpha_{1 k}^{\dagger} \beta_{2 k}-\beta_{1 k}^{\dagger} \alpha_{2 k}\right)\right)+ \text { h.c. }\right]\right\}
\end{aligned}
$$

with

$E_{k}=\left[4 t_{\|}^{2} \cos ^{2}(k a)+\Delta_{0}^{2} \sin ^{2}(k a)\right]^{1 / 2}$.

The full Hamiltonian can now be diagonized for the case of $u_{1}=u_{2}$ (parallel ordering, see Fig. 3(b)) and $u_{1}=-u_{2}$ (anti-parallel ordering, see Fig. 3(c)). We find that the anti-parallel ordering case is always energetically favorable. Below we set $u_{1}=u$, and $u_{2}=-u$, then calculate and minimize the ground state energy with respect to $u$ and $w$. The energy dispersion of the two bands $\varepsilon_{v \pm}(k)$ below the Fermi level are

$$
\begin{aligned}
\varepsilon_{v \pm}= & -\left[\left(\frac{t_{0}^{2}}{4}+4 t^{\prime 2}\right)-\left(4 t^{\prime 2}-\Delta_{0}^{2}\right) \sin ^{2}(k a)\right] \\
& \pm \frac{t_{0}}{2}
\end{aligned}
$$

where $t^{\prime} \equiv t_{0}-2 \alpha w$, and gap parameter $\Delta_{0} \equiv 4 \alpha u$. The two band above the Fermi level $\varepsilon_{\mathrm{c} \pm}(k)$ are equal to $-\varepsilon_{v \pm}(k)$. The band gap (at $k a= \pm \pi / 2$ ) is $E_{\mathrm{g}}=2 \sqrt{\Delta_{0}^{2}+\frac{t_{0}^{2}}{4}}-t_{0}$

The total energy per electron turns out to be

$\bar{\varepsilon}=-\frac{4}{\pi} \sqrt{4 t^{\prime 2}+\frac{t_{0}^{2}}{4}} E(Z)+\frac{K}{2}\left(4 u^{2}+4 w^{2}\right)$,

with

$$
Z=\frac{4 t^{\prime 2}-\Delta_{0}^{2}}{4 t^{\prime 2}+t_{0}^{2} / 4}
$$

In the following we adopt the physical parameters for polyacetylene and search for $u_{0}$ and $w_{0}$ that minimizes $\bar{\varepsilon}$. Surprisingly, the minimal value of $\bar{\varepsilon}$ occurs at $u_{0}=0$ and $w_{0}=-0.119 \AA$. The fact that $u_{0}=0$ at the minimum appears to be a general property of the function $\bar{\varepsilon}(u, w)$, and does not depend on the particular choice of the physical parameters. The dimerization order parameter $u_{0}=0$ implies $\Delta_{0}=0$ and $E_{\mathrm{g}}=0$. Polyacene, therefore, should be classified as a "gapless semiconductor". Its band structure and density of state are plotted in Fig. 4. Since the density of state near the Fermi level $\left(\varepsilon_{\mathrm{F}}=0\right)$ is singular, many properties are expected to be different from the conventional metals or semiconductors. The interesting point here is that the consideration of bond length alternation parameter $w$ makes our result qualitatively different from the others. In fact, a nonzero $u_{0}$ and $E_{\mathrm{g}}$ do develop if we force the bond length order parameter $w$ to be zero. 

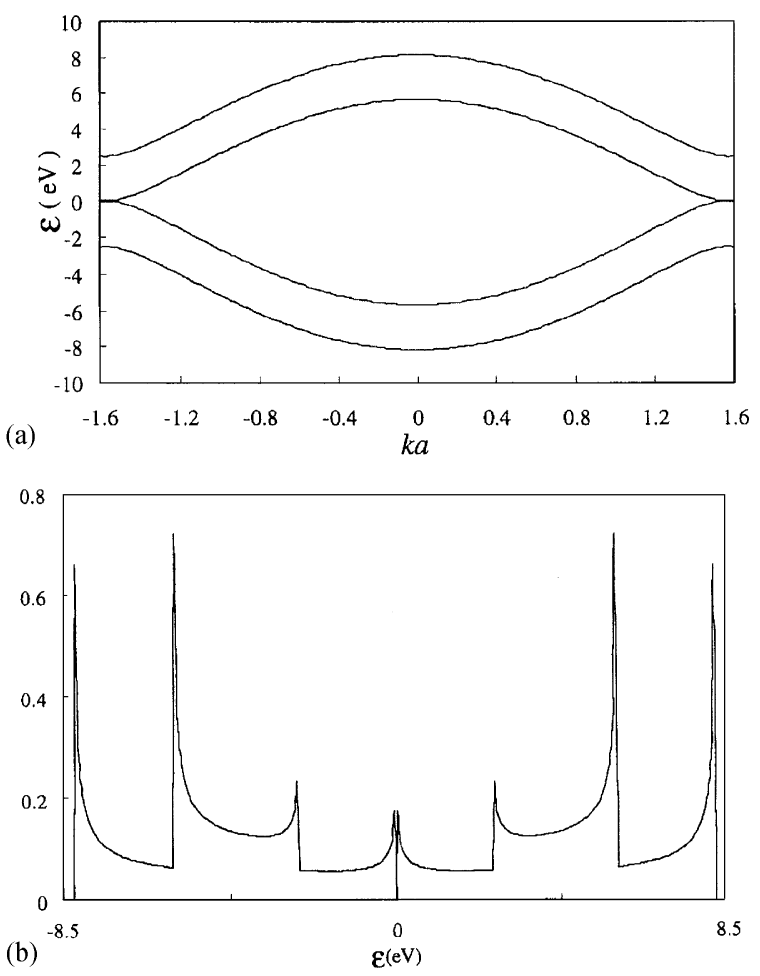

Fig. 4. The band structure (a) and the density of states (arbitrary unit) (b) of the ground state of polyacene.

\subsection{High DC conductivity}

Doping-induced quasi-particles with strong lattice distortion are widely believed to be the main charge carriers in most conducting polymers. For a gapless polymer, the quasi-particles like solitons or polarons are unstable compared with the Bloch electron, whose energy can be arbitrarily small. One then expects that the transport properties of polyacene to be very different from other more conventional conducting polymers. The carriers contributing to the conductivity include electrons (conduction band) and holes (valence band). For both bands, the electric conductivity is

$\sigma=-N_{\mathrm{c}} \int_{0}^{\varepsilon_{0}} \sigma(\varepsilon) \frac{\partial f_{0}}{\partial \varepsilon} \mathrm{d} \varepsilon$,

where $N_{\mathrm{c}}$ is the number of polyacene chains per $\mathrm{cm}^{2}$, and

$\sigma(\varepsilon)=e^{2} v(\varepsilon)^{2} \tau(\varepsilon) \rho(\varepsilon)$.
Here $f_{0}, \tau, \rho$ are the Fermi distribution function, carrier relaxation time, and density of state, respectively. The carrier velocity $v$ is equal to $(1 / \hbar) \partial \varepsilon / \partial k$. We apply a model developed by Conwell [14] for one-dimensional organic semiconductors to calculate the relaxation time $\tau$. The interaction Hamiltonian of the electrons with LA phonons is

$H_{\mathrm{e}-\mathrm{ph}}=N^{-1 / 2} \sum_{q} g_{k, q} c_{k+q}^{\dagger} c_{k}\left(b_{q}+b_{-q}^{\dagger}\right)$,

where $b_{q}$ is the annihilation operator for a phonon with wave vector $q$, and $c_{k}$ is the annihilation operator of a conduction or valence state with wave vector $k$. The electron-phonon coupling constant $g_{k, q}$ is

$g_{k, q}=\mathrm{i} 4 \alpha\left(\frac{\hbar}{2 M \omega_{q}}\right)^{1 / 2}[\sin (k+q) a-\sin k a]$.

$M$ is the total mass of the unit cell, and $\alpha$ is the electron-lattice coupling constant in the SSH model. In one-dimensional system, scattering basically take place between $\pm k$ and $\mp k$ because the acoustic mode scattering is essentially elastic. In such cases we have

$g_{k, q}=\mathrm{i} 8 \alpha\left(\frac{\hbar}{2 M \omega_{q}}\right)^{1 / 2} \sin k a$.

The relaxation time $\tau$ can be expressed as $1 / \tau=$ $1 / \tau_{\mathrm{em}}+1 / \tau_{\mathrm{abs}}$. The subscripts "em" and "abs" indicate phonon emission and absorption, respectively. The formula for them are derived by Conwell

$$
\begin{aligned}
\frac{1}{\tau_{\mathrm{em}}}= & \frac{2 \pi}{\hbar} g_{k}^{2} \Theta\left(\varepsilon-\hbar \omega_{2 k}\right) \rho\left(\varepsilon-\hbar \omega_{2 k}\right)\left(n_{2 k}+1\right) \\
& \times\left(\frac{1-f_{0}\left(\varepsilon-\hbar \omega_{2 k}\right)}{1-f_{0}(\varepsilon)}\right), \\
\frac{1}{\tau_{\mathrm{abs}}}= & \frac{2 \pi}{\hbar} g_{k}^{2} \Theta\left(\varepsilon_{0}-\left(\varepsilon+\hbar \omega_{2 k}\right)\right) \rho\left(\varepsilon+\hbar \omega_{2 k}\right) n_{2 k} \\
& \times\left(\frac{1-f_{0}\left(\varepsilon+\hbar \omega_{2 k}\right)}{1-f_{0}(\varepsilon)}\right),
\end{aligned}
$$


where $\Theta$ is the step function. The density of state $\rho(\varepsilon)$ for polyacene is

$$
\begin{aligned}
\rho(\varepsilon)= & \frac{1}{\pi a} \frac{\varepsilon+t_{0} / 2}{\left(t_{0}-2 \alpha \omega_{0}\right)^{2}} \\
& \times\left(\sin \left[2 \cos ^{-1}\left[\frac{\sqrt{\varepsilon^{2}-\varepsilon t_{0}}}{2\left(t_{0}-2 \alpha \omega_{0}\right)}\right]\right]\right)^{-1} .
\end{aligned}
$$

Substituting Eqs. (39) and (40) back into Eq. (35) and including both the electrons and the holes, we obtain the electric conductivity $\sigma$. Because of its peculiar gapless band structure, one expects that under low doping level the thermal electron will dominate, and the conductivity is independent of the doping concentration, contrary to the conventional conducting polymers like polyacetylene. Fig. 5 shows our numerical results of the conductivity of polyacene as a function of dopant level at two opposite temperature limits. Results for polyacetylene, a typical one-dimensional semiconductor with a band gap of $1.4 \mathrm{eV}$, are also shown for comparison. One sees that the conductivity of polyacene remains unchanged for $T=300 \mathrm{~K}$. For $T=0.1 \mathrm{~K}$, its conductivity keeps constant until the dopant concentration reaches as high as $10^{-4}$ carriers/Å. For polyacetylene, the conductivity increases linearly with the dopant concentration. In Fig. 6, we show the temperature dependency of the conductivity. Instead of an exponential growth, the conductivity is proportional to the temperature below 200 K. Sommerfeld expansion of Eq. (34) in $T$ actually leads to a positive linear term and a negative quadratic term.

\section{Conclusion}

Dimerization has been believed to be the only effect the electron-lattice coupling causes to the lattice configuration for the ground state of conjugated polymers. We found that the chain length contraction also happens simultaneously. The contraction leads to a new bond length order parameter, which varies in space around quasi-particles like solitons and polarons. A set of self-consistent equations is derived and solved numerically for the order parameter. The resulting electron spectrum

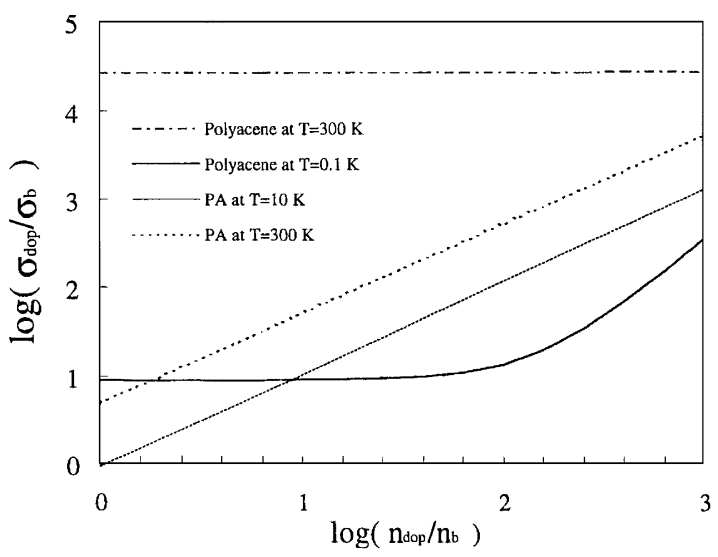

Fig. 5. Comparison of the DC conductivity $(\sigma)$ of polyacene and trans-PA under various dopant concentrations. The dopant concentration $n_{\mathrm{b}}=10^{-6} / \AA$, and $\sigma_{\mathrm{b}}$ denotes the conductivity of trans-polyacetylene for $n_{\mathrm{dop}}=n_{\mathrm{b}}$ at $300 \mathrm{~K}$.

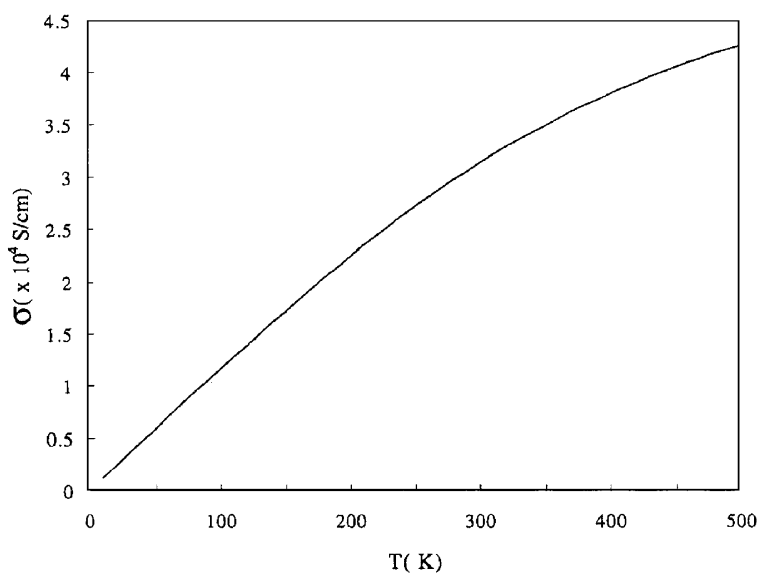

Fig. 6. The temperature $(T)$ dependence of the DC conductivity $(\sigma)$ of polyacene without doping is shown. One can see that $\sigma$ is almost linear in $T$ below about $200 \mathrm{~K}$.

for polaron is quite different from the case that only dimerization is considered. The polaron mobility, which is determined by the particular form of lattice distortion around the carrier, will also be modified significantly. By including the bond length order parameter, we also predict that the ground state configuration of polyacene is a gapless semiconductor, contrary to many previous suggestions that it has a gap around $0.4 \mathrm{eV}$. Due to such 
a peculiar band structure, polyacene is a much better conductor compared with other conducting polymers. The conductivity can be as high as $4 \times 10^{4} \mathrm{~s} / \mathrm{cm}$ without doping, which is at least oneorder of magnitude larger than the typical conducting polymers like polyacetylene. Of course our calculations apply to the intrachain transport only. The actual value of the conductivity is limited by the interchain hopping and depend on the level of disorder, which we do not consider in this work. In addition, a small gap may develop purely due to the Coulomb interaction. Our prediction of a high conductivity should still hold qualitatively even in such case.

\section{Acknowledgements}

The authors are grateful for the support by the National Science Council of Taiwan under contract No. NSC86-2112-M-009-001. The hospitality of the National Center for Theoretical Sciences in Taiwan is also appreciated.

\section{References}

[1] A.J. Heeger, S. Kivelson, J.R. Schrieffer, W.P. Wu, Rev. Mod. Phys. 60 (1988) 781.

[2] H.G. Kiess (Ed.), Conjugated Conducting Polymers, Springer, Berlin, 1992.

[3] R.E. Peierls, Quantum Theory of Solids, Clarendon press, Oxford, 1959.

[4] T. Kennedy, E.H. Lieb, Phys. Rev. Lett. 59 (1987) 1309.

[5] W.P. Su, J.R. Schrieffer, A.J. Heeger, Phys. Rev. B 22 (1980) 2099.

[6] H. Takayama, Y.R. Lin-Liu, K. Maki, Phys. Rev. B 21 (1980) 2388.

[7] D.K. Campbell, A.R. Bishop, Nucl. Phys. B 200 (1982) 297.

[8] J.Z. Ruan, M.H. Litt, in: M.J. Bowden, S.R. Turner (Eds.), Polymers for High Technology, ACS, Washington DC, 1987.

[9] S. Canuto, Jose D' Albuquerque e Castro, F.J. Paixao, Electronic Structure of Atoms and Molecules and Solids, World Scientific, Singapore, 1990.

[10] L. Salem, H.C. Longuett-Hinggins, Proc. Roy. Soc. London Ser. A 235 (1960) 435.

[11] R. Hoffman Whangbho, R.B. Woodward, Proc. Roy. Soc. London Ser. A 366 (1979).

[12] K. Tanaka, K. Ozheki, S. Naukai, T. Yamabe, H. Shirakawa, Phys. Solids 44 (1983) 1069.

[13] A.L.S. Rosa, C.P. de Melo, Phys. Rev. B 38 (1988) 5430.

[14] E.M. Conwell, Phy. Rev. B 22 (1980) 1761. 